Abstracta Iranica Abstracta Iranica

Revue bibliographique pour le domaine irano-aryen

Volume 40-41 | 2019

Comptes rendus des publications de 2017-2018

\title{
Kaan Iren, Çiçek Karaöz, Özgun Kasar (eds.). Persler: Anadolu'da Kudret ve Görkem - The Persians. Power and Glory in Anatolia
}

\section{Sébastien Gondet}

\section{(2) OpenEdition}

\section{Journals}

Édition électronique

URL : http://journals.openedition.org/abstractairanica/49182

DOI : 10.4000/abstractairanica.49182

ISBN : 1961-960X

ISSN : 1961-960X

Éditeur :

CNRS (UMR 7528 Mondes iraniens et indiens), Éditions de l'IFRI

Référence électronique

Sébastien Gondet, « Kaan Iren, Çiçek Karaöz, Özgun Kasar (eds.). Persler: Anadolu'da Kudret ve Görkem

- The Persians. Power and Glory in Anatolia », Abstracta Iranica [En ligne], Volume 40-41 | 2019,

document 46, mis en ligne le 30 octobre 2019, consulté le 24 avril 2021. URL : http://

journals.openedition.org/abstractairanica/49182; DOI : https://doi.org/10.4000/abstractairanica. 49182

Ce document a été généré automatiquement le 24 avril 2021.

Tous droits réservés 


\title{
Kaan Iren, Çiçek Karaöz, Özgun Kasar (eds.). Persler: Anadolu'da Kudret ve Görkem - The Persians. Power and Glory in Anatolia
}

\author{
Sébastien Gondet
}

\section{RÉFÉRENCE}

Kaan Iren, Çiçek Karaöz, Özgun Kasar (eds.). Persler: Anadolu'da Kudret ve Görkem - The Persians. Power and Glory in Anatolia. Istanbul: Yap1 Kredi Yayınları, 2017, 424p. Bilingue turc-anglais. (Anatolian Civilizations Series 6)

1 Ce volume est le sixième d'une collection de monographies portant sur les différentes civilisations antiques qui ont cohabité et se sont succédées en Anatolie. Née de la volonté d'une maison d'édition basée à Istanbul, cette série se propose de mettre à disposition du grand public des synthèses richement illustrées et rédigées par des historiens, historiens de l'art et des archéologues intégrant les récentes avancées dans leur domaine respectif. En plus du présent volume portant sur la période achéménide, sont également parues des synthèses sur les Hittites, les Assyriens, les Lyciens, les Phrygiens, l'Urartu et enfin la cité de Pergame.

2 Ce volume dédié aux Perses en Anatolie rencontrera certainement l'intérêt de la communauté scientifique travaillant sur l'Achéménide pour au moins deux raisons. D'une part, il rassemble des contributions de spécialistes turcs et internationaux en version bilingue turc-anglais. Ainsi les lecteurs non-turcophones trouveront dans ce volume des données et des synthèses qui n'étaient jusqu'à présent disponibles qu'en turc. D'autre part, les illustrations sont de très bonne qualité et plusieurs sont inédites. L'ensemble des articles offre un tour d'horizon très complet de la période achéménide dans l'ensemble de la péninsule anatolienne. Tous se concluent par une riche 
bibliographie. Ce volume constitue donc une porte d'entrée idéale à tous ceux qui s'intéresseraient à l'Anatolie achéménide.

3 Il nous semble pertinent de détailler le sommaire de ce volume et de présenter cidessous un très bref résumé de chacune des 22 contributions.

- Tomris Bakır. Tracing the Persians' footsteps in Anatolia: 2-9. Cette introduction traite de la naissance d'une culture matérielle perso-anatolienne en l'illustrant à partir des principaux résultats des fouilles du site de Daskyleion que l'A. a dirigées de 1988 à 2008.

5 - Pierre Briant. Who are the Persians ?: 12-19. Cet article présente les principales sources textuelles disponibles pour étudier l'histoire de la période achéménide et définit à grands traits les structures de l'empire et le rôle des Perses comme ethno-classe dominante.

6 - Fahri Işık. Anatolia before the Persians: 24-49. L'A. présente les principales entités politiques et cultures qui se sont succédées et ont cohabité en Anatolie au cours de la première moitié du $1^{\text {er }}$ millénaire avt. J.-C. Il souligne en plusieurs occasions la continuité, sur le long terme, de traditions louvites en Anatolie.

7 - Frédéric Maffre. The Anatolian people under the Persian rule: 52-63. Cet article détaille d'abord la géopolitique de l'Anatolie au cours de la période achéménide. L'A. dresse ensuite la liste des données matérielles qui démontrent l'émergence au sein des élites d'un art perso-anatolien mais constate surtout la continuation des cultures matérielles préexistantes.

8 - Eric. A. Raimond. Gods and peoples in Persian Anatolia: the case of Lycian deities: 66-74. En Lycie, mis à part quelques timides indices de cultes "exogènes ", l'A. constate une continuité, au cours de la période achéménide, des croyances et pratiques religieuses préexistantes. Cette démonstration s'appuie en grande partie sur une description et une interprétation de l'inscription trilingue du Létôon de Xanthos.

9 - Sevgi Sarıkaya, Murat Arslan. Persians in Anatolia: war, rebellion and diplomacy: 78-99. Basé essentiellement sur les textes grecs, on trouvera ici un récit historique des grands évènements qui ont marqué les relations entre le pouvoir achéménide et les satrapies anatoliennes ainsi que les cités-états grecques.

10 - Oğuz Tekin. Money and trade during the Persian period in Anatolia: 102-133. Cet article constitue une synthèse minutieuse des données numismatiques disponibles pour la période achéménide en les détaillant par province. Il apparaît que la domination perse a favorisé la frappe de monnaies et les échanges entre les grandes cités anatoliennes.

11 - Murat Arslan. The Asia Minor campaign of Alexander and the Battle of Issus. Portrait of a battle that shaped ancient history : 136-159. Utilisant sources antiques et iconographiques, l'A. retrace les étapes de la conquête de l'Asie Mineure par les armées d'Alexandre.

12 - Suat Ateşlier. Architecture during the Persian period in Anatolia: 162-171. Les données prises en compte se concentrent sur les monuments funéraires commandités par les satrapes ou les membres des élites locales. Ce sont les seules réalisations architecturales « perses » dont on conserve des témoins bien attestés.

13 - Hülya Bulut. Earthly paradises: Persian Gardens and Paradeisoi: 174-183. Se basant très majoritairement sur les descriptions des paradis anatoliens par les auteurs grecs, cet article traite de leurs fonctions et de leur symbolique

14 - Gürcan Polat. Persian influence in stone carving in Anatolia: 186-201. Issus de découvertes fortuites et dispersés dans divers musées de Turquie, cet article décrit les exemples les 
mieux conservés et les plus illustratifs de bas-reliefs funéraires (stèles, blocs architecturaux, sarcophages) de style perso-anatolien.

15 - Figen Çevirici-Coşkun. The Polyxena sarcophagus: a Persian aristocrat's sarcophagus? 204-221. Mis au jour au cours de la fouille de sauvetage d'un tumulus au nord-ouest de la Turquie, dans la province de Çanakkale, le sarcophage de Polyxène est décoré de remarquables bas-reliefs de style ionien. Des éléments de contexte archéologique caractéristiques, d'après l'A., des pratiques funéraires de la période achéménide en Anatolie permettent d'attribuer ce sarcophage à un noble perse.

16 - Sedef Çokay-Kepçe. Achaemenid terracotta bowls and phiale found in Anatolia: overview: 224-235. Les bols carénés et phiales achéménides sont souvent considérés comme des marqueurs de l'apparition de nouvelles pratiques commensales, inspirées de celles des Perses, au cours de la seconde moitié du $1^{\text {er }}$ millénaire. L'A. liste les sites et les contextes où ont été mis au jour des exemples de cette vaisselle caractéristique. Selon l'A., les données archéologiques suggèreraient une apparition juste antérieure à la période achéménide.

17 - Inci Delemen. Prestige objects (insignia) with Persian influences in Anatolia: 238-251. Les productions de prestige en métal (petit mobilier, vaisselles, bijoux) retrouvées pour certains au cours des fouilles de plusieurs tumuli (région d'Ushak en Lydie ou site d'Ikiztepe plus au nord) démontrent la diffusion d'un art de cour perse.

18 - Deniz Kaptan. Seals from Achaemenid Anatolia: 254-267. Les découvertes de sceaux ou d'empreintes de sceaux d'époque achéménide sont particulièrement nombreuses comparées aux époques antérieures. Le corpus intègre des sceaux royaux achéménides, à Daskyleion, mais on note surtout la récurrence de motifs plutôt mésopotamiens.

19 - Margaret C. Miller. The Persian presence in Anatolia - An Athenian perspective: 270-280. Certains vases peints produits en Grèce représentent les Perses d'Asie Mineure. L'A. souligne une évolution entre les représentations les plus anciennes à valeur quasi ethnographique et celles plus récentes soulignant l'adoption d'attributs perses par les élites anatoliennes.

20 - Adnan Diler. Iconography in Anatolian-Persian art: tradition, political realism and paradox: 284-305. L'A. liste les thèmes récurrents de l'iconographie perso-anatolienne: processions, banquets, combats, audiences, rituels. Cet article complète les précédents car il considère le corpus iconographique dans son ensemble et on trouvera par exemple de longs commentaires sur les peintures sur bois du tumulus de Tatarl.

21 - Nicholas Cahill. The Satrapy of Lydia: 308-329. Cet article offre un tour d'horizon bien documenté des données sur l'occupation achéménide à Sardes et plus largement en Lydie. Malgré des données encore parcellaires, il apparait très nettement que la période achéménide est marquée par d'importants changements dans l'organisation urbaine et territoriale.

22 - Kaan Iren, Handan Yıldızhan. Persian Dascyleum: a satrapal center in the Southern Marmara region: 332-347. On trouvera dans cet article une synthèse des données archéologiques, incluant celles issues des campagnes fouilles en cours dirigées par les A., sur la période achéménide à Daskyleion. Plusieurs des illustrations proviennent des archives inédites de la mission et témoignent des importantes découvertes effectuées lors des fouilles de deux tumuli funéraires situés non loin du site.

23 - Olivier Can Henry. Hecatomnus, son of Hyssaldomus: a unicum in Persian history: 350-365. Le règne d'Hecatomnus, dynaste de Carie désigné satrape par le pouvoir achéménide, 
n'a laissé qu'une poignée de témoins archéologiques. Une grande partie de cet article étudie les différentes hypothèses d'identification de sa tombe. Un long développement est consacré aux fouilles, encore largement inédites, de la tombe d'Uzunyuva à Milas.

24 - F. Eray Dökü. Persian traces in the West Black Sea region: 368-387. Dans la province de Paphlagonia, les vestiges achéménides sont rares. Comme ailleurs en Anatolie, ils consistent essentiellement en des vestiges funéraires : stèles décorées de bas-relief ou tombes rupestres présentant un grand nombre de similitudes avec celles de Persépolis.

25 - Stuart Blaylock. The Achaemenid level at Tille Höyük on the Euphrates and other evidence for Achaemenid archaeology in South-East Anatolia: 390-409. Les fouilles d'un grand bâtiment à Tille Höyük et plus récemment $d u$ cimetière de Gre Amer ont mis au jour une architecture et du matériel d'époque perse plutôt caractéristique du nord de la Mésopotamie. L'A. propose également une intéressante réflexion sur la capacité des archéologues à définir correctement les assemblages de l'époque perse souvent considérée comme un « dark age ».

\section{AUTEURS}

\section{SÉBASTIEN GONDET}

UMR 5133 CNRS-Université de Lyon 